\title{
Analysis of microbial community and pollutants removal performance in the simulated reaction pool by immobilized microbe technology
}

\author{
Qing Fang ${ }^{1}$, Hai-fang Tang ${ }^{2}$, Ping Xian, ${ }^{1, *}$, and Jun-cen Bai ${ }^{1}$ \\ ${ }^{1}$ College of Environment of Guangxi University, Nanning 530004, China \\ ${ }^{2}$ College of Chemistry and Chemical Engineering, Hunan University, Hunan 410082, China
}

\begin{abstract}
In this lab-scale simulation, the performance of aerated immobilized microbe (IM) in in-situ pond sedimentation remediation, and the microbial community was studied. It is known for the results that total organic matter (TOM), total nitrogen $(\mathrm{TN})$ and total phosphorus (TP) in the sediment can be effectively reduced by aerated immobilized microbe technology. The polluted raw water, classified as inferior Class- $V$ according to the surface water environment quality standard (GB3838-2002), upgraded to Class-III in 30-days remediation with TOM, TN and TP removal rates of 33.35\%, 43.35\% and 23.20\%, respectively. In contract, the control group without the assistant of aerated immobilized microbe still remains at Class-IV. High-throughput sequencing analysis reveals that immobilized microbe technology could improve the species diversity and abundance of the flora, and enhance the similarity between the sediment and the overlying water in the system. The dominant bacteria of the overlying water were Perlucidibaca, Limnohabitans, Bacillus, Hydrogenophaga, Mycobacterium etc. And the dominant bacteria of the sediment were Methanolinea, Methanoregula, Candidatus Competibacter, Methanosaeta etc. Total phosphorous, certified by the redundancy analysis, is the key environmental factor of microbial community structure in both overlying water and sediment.
\end{abstract}

\section{Introduction}

In recent years, IM has become a research hotspot, the technology degrade pollutants through oxidation, reduction, hydrolysis of the IM. Compared with the traditional biotechnology, the technology have many advantages, such as higher reaction rates due to increased cell densities, possibilities for regenerating the biocatalytic activity of IM structures, ability to conduct continuous operations at high dilution rate without washout, easier control of the fermentation process, long-term stabilization of cell activity, higher specific product yields etc. ${ }^{[1]}$ IM technology has good effect on both water body and sediment, and the community structure and population diversity of microorganisms in the system can reflect the running status of the system to a certain extent. Therefore, the study of dominant populations, community biodiversity in the system is of great practical significance to clarify the microbial structure and improve the efficiency of water quality purification in the system. In this paper, IM technology was used to simulate the in situ restoration of lake sediments. The effect of pollutant removal in the reaction tank was studied, and the overlying water and sediment samples were obtained during the stable operation period to analysis the microbial community composition and structure by high-throughput sequencing technique. This is of great significance to optimize the operation of the reactor and improve the treatment effect. And can provide reference for the practical application of IM technology in repairing the sediment in the lake.

\section{Materials and methods}

\subsection{Experimental setup and operation}

The experimental setup is shown in Fig. 1a, with plexiglas column longitudinal simulation of natural lake water and sediment to carry out experiments. The reaction pool used in the study has a dimension of 100 $\mathrm{cm}$ height $\times 20 \mathrm{~cm}$ length $\times 20 \mathrm{~cm}$ width. Microporous aeration device and immobilized microbial carrier column were suspended in the reaction cell, the immobilization rate of immobilized microbial carrier was about $1 \%$, and the carrier column was filled with a variety of ceramic as the medium. Immobilized microbial carrier filling rate of about $1 \%$, the carrier column filled with immobilized microbial particles, which was immobilized on various dominant species by embedding

* Corresponding author: xianping@gxu.edu.cn. 
method using ceramic as medium (including aerobic, aerobic and anaerobic bacteria, and aerobic) as shown in Fig. 1b. Generally microorganisms remain dormant in the carrier, but when the carrier is placed in the waste water and supplied with oxygen, microbes are activated by the nutrients in the water, continuous breeding, so that the entire treatment system can maintain a high concentration of microbial content.

The experiment was carried out under optimum conditions (the immobilized microbial carrier column is located at the muddy water interface with overlying water DO of $5.5 \sim 6.5 \mathrm{mg} / \mathrm{L}$ and $\mathrm{pH}$ 7) which has been determined by experiment and marked as IM group. And set the blank control group does not carry out any processing, marked as CON group at the same time.

The water and sediment used in this study were taken from Guangxi University campus lake, and filled with sediment (a depth of $20 \mathrm{~cm}$ ) and raw water (a depth of 60 $\mathrm{cm})$ in each reaction pool.

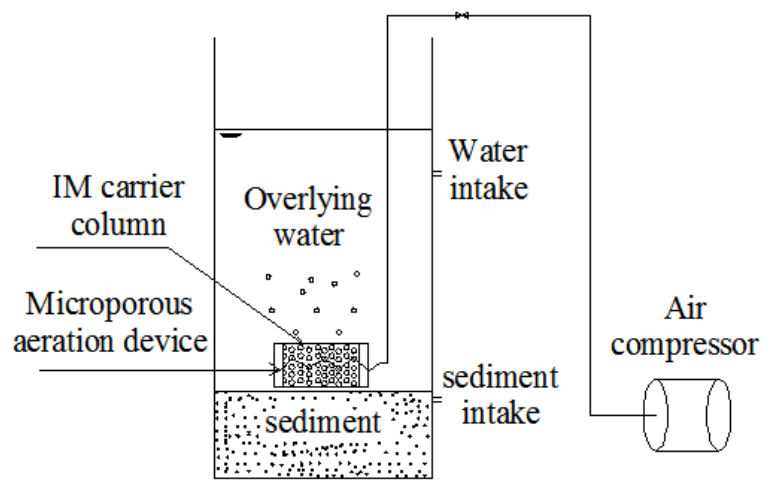

a

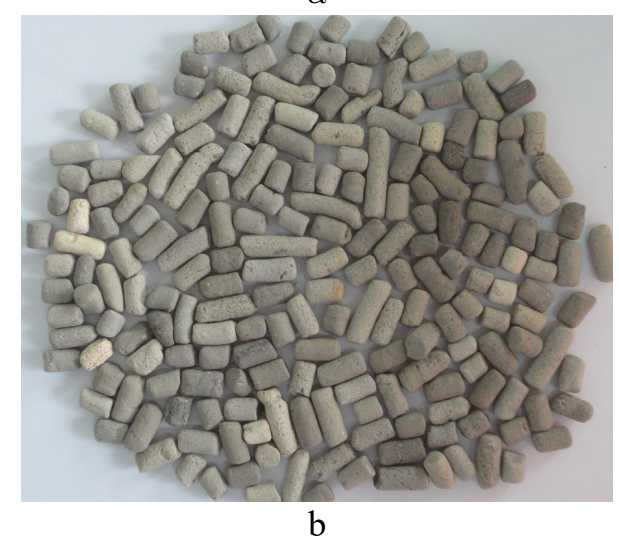

Fig.1. Experimental equipment and immobilized microbe carriers

\subsection{Sampling and analysis}

The study lasted for 30 days. During the experimental period water samples $(200 \mathrm{~mL})$ were collected every day from each tank at approximately 9:00am and filtered through 0.45 um $\mathrm{GF} / \mathrm{C}$ filter paper under vacuum pressure and analysed chemical oxygen demand(COD) by microwave digestion and spectrophotometrically for ammonia nitrogen $\left(\mathrm{NH}_{4}{ }^{+}-\mathrm{N}\right)$ and $\mathrm{TN}, \mathrm{TP}$, following 'Standard methods for the examination of water and wastewater'. And sediment samples were taken to analyse every $15 \mathrm{~d}$. According to standard methods all samples were transferred immediately to the lab and analysed immediately for TOM, TN, TP.

\subsection{DNA extraction}

At the end of the experiment, water samples of the experimental group, the control group and the original group (untreated) were filtered through $0.22 \mathrm{um}$ membrane filters and were extracted with OMEGA Water DNA Kit,D5525, marked as: IMW, CONW, ORIW respectively. The sediment samples were then collected at four equidistant positions on the diagonal of each reactor and mixed proportional, and then extracted with OMEGA Soil DNA Kit, D5625, marked as: IMS, CONS, and ORIS respectively. Then, all extracted DNA samples from water and sediment were sent to Novogene Bioinformatics Technology Co.Ltd for sequencing.

\subsection{Evaluation of richness and diversity}

Mothur software was used to analyse alpha diversity ${ }^{[2]}$. Community diversity in samples was estimated by Shannon-Wiener indices and rarefaction curves were used to assess species richness. The total number of species in samples (an exponent of the OTU number) was estimated with Chaol ${ }^{[3]}$. Sampling coverage was used to evaluate the authenticity of sequenced data.

\subsection{Canoco analysis}

The relationship among designated microbial communities in samples and water quality indicators was analysed by multivariate statistical analysis software (Canoco for Windows 4.5). Program WcanoImp of the Canoco for Windows 4.5 software package was used to generate spe.dta and env.dta files and detrended correspondence analysis (DCA) was carried out. Canonical correspondence analysis (CCA) or redundancy analysis (RDA) was used for ordination according to gradient lengths (RDA was used when gradient lengths was less than 3; RDA or CCA was used when gradient lengths was between 3 and 4; CCA was used when gradient lengths was more than 4) to address the relationship between microbial communities and water quality indicators.

\section{Results and discussion}

\subsection{Pollutants removal}

\subsubsection{Organic matter removal}

The relationship between water and sediment quality parameters and microbial communities in each pool was investigated by monitoring the quality parameters of water and sediment samples collected during the whole experiment. The fluctuations of $\mathrm{COD}_{\mathrm{Mn}}$ in overlying water and TOM in sediment throughout the experimental period were shown in Fig. 2. Under the action of 
immobilized microbe (IM), organic matters degradation removal efficiency was much higher than that of non-immobilized microorganisms (CON). As shown in Fig. 2a, the $\mathrm{COD}_{\mathrm{Mn}}$ concentration of overlying water in IM system was below $6 \mathrm{mg} / \mathrm{L}$ during the experiment period, which can comply with the Class III of Surface Water Environment Quality Standard (GB3838-2002) in china. However, in CON system, the $\mathrm{COD}_{\mathrm{Mn}}$ concentration of overlying water ranged between $8 \mathrm{mg} / \mathrm{L}$ and $12 \mathrm{mg} / \mathrm{L}$. As shown in Fig. 2b, the TOM removal rate of sediment in IM system $(33.35 \%)$ was $11.15 \%$ higher than that of CON system $(22.20 \%)$.

The significant decrease of organic matter concentration in IM system was observed due to the microbes in IM system are activated and multiply, under the action of microbial metabolism, organic matter was removed. Moreover, the DO concentration of IM system was kept between $5.5 \mathrm{mg} / \mathrm{L}$ to $6.5 \mathrm{mg} / \mathrm{L}$ all the time, the high DO concentration also can promote the removal of organic matters. It has been reported that biodegradation and aerobic conditions are main factors contributing to the removal of organic matter. ${ }^{[4]}$

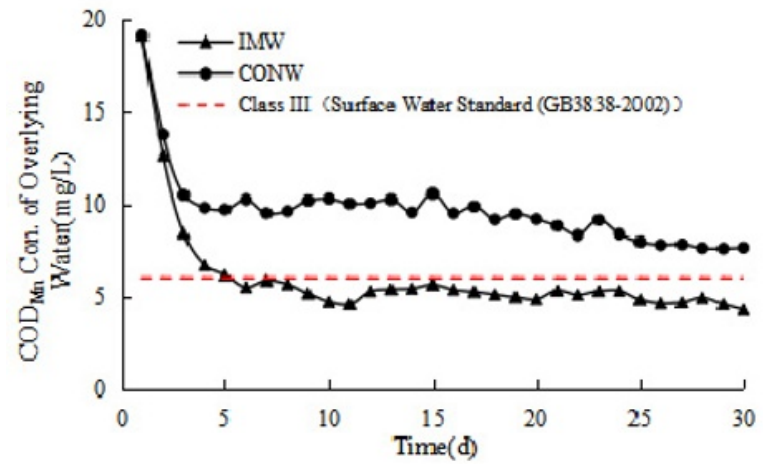

a

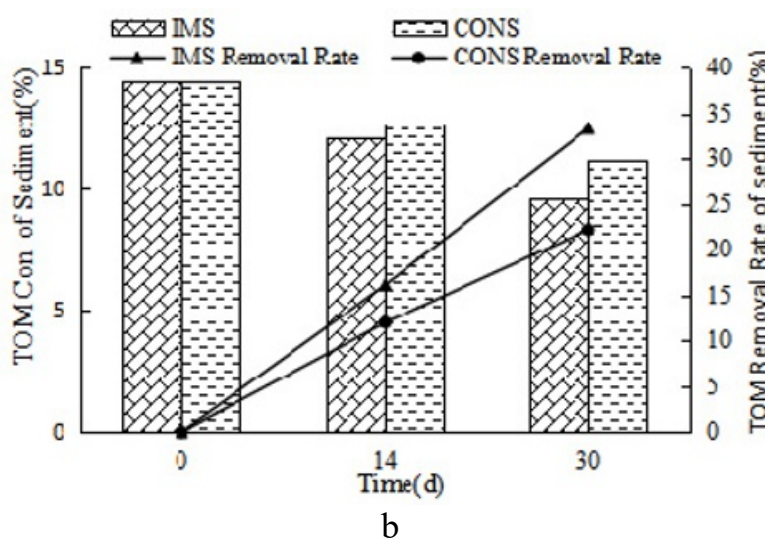

Fig.2. Effect of immobilized microorganism on carbon pollutants

\subsubsection{Nitrogen removal}

The fluctuations of $\mathrm{NH}_{3}-\mathrm{N}, \mathrm{TN}$ in overlying water and $\mathrm{TN}$ in sediment throughout the experimental period are shown in Fig. 3. Under the action of immobilized microbe, nitrogen degradation removal efficiency in IM system was much higher than that of CON system. As shown in Fig. 3a, the NH3-N concentration of overlying water in IM system was below $0.15 \mathrm{mg} / \mathrm{L}$ during the experiment period, which can comply with the Class I of
Surface Water Environment Quality Standard (GB3838-2002) in china. However, in CON system, the $\mathrm{NH} 3-\mathrm{N}$ concentration of overlying water ranged between $1 \mathrm{mg} / \mathrm{L}$ and $2 \mathrm{mg} / \mathrm{L}$. And the $\mathrm{TN}$ concentration of overlying water in IM system was below $1 \mathrm{mg} / \mathrm{L}$ during the experiment period, which can comply with the Class III of Surface Water Environment Quality Standard (GB3838-2002) in china. However, in CON system, the TN concentration of overlying water ranged between 2 $\mathrm{mg} / \mathrm{L}$ and $6 \mathrm{mg} / \mathrm{L}$. As shown in Fig. 3b, the TN removal rate of sediment in IM system $(43.66 \%)$ was $12.67 \%$ higher than that of non-IM system $(30.99 \%)$.

The carrier we used in this experiment has different pore sizes. A gradient of DO is formed inside and outside the carrier under the diffusion of oxygen. The diffusion in the reactor is limited, due to the limited ability of oxygen permeation, resulting in the highest DO concentration on the outer surface of the carrier which was mainly distributed with heterotrophic aerobic bacteria, nitrosated bacteria and nitrifying bacteria and an anaerobic zone in the carrier which can provide conditions for denitrification of anaerobic denitrifying bacteria. ${ }^{[5]}$ In summary, the IM group has better removal effect on nitrogen containing pollutants.
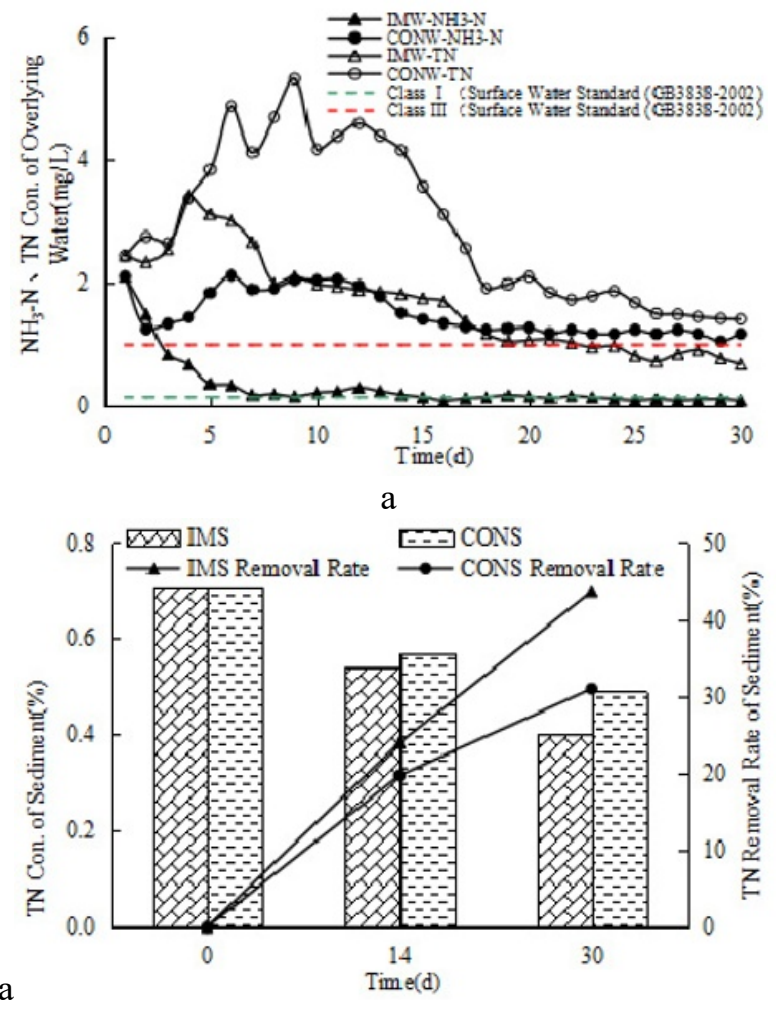

b

Fig.3. Effect of immobilized microorganism on nitrogen pollutants

\subsubsection{Phosphorus removal}

The fluctuations of TP in overlying water and TP in sediment throughout the experimental period are shown in Fig. 4. Different from $\mathrm{NH}_{3}-\mathrm{N}$ and $\mathrm{TN}$ removal rate, there was no significant difference in TP. TP concentration of the overlying water in both groups was kept at a low level, which can comply with the Class III 
of Surface Water Environment Quality Standard (GB3838-2002) in china. And the removal rate of TP in the sediment is not high in both groups either. Which may because of the phosphorus element cannot become gas to leave the reaction system, it can only be transferred between the water body, sediment and microorganisms, when the microorganisms die, and the phosphorus element will rapidly return to the sediment or water again ${ }^{[4]}$.

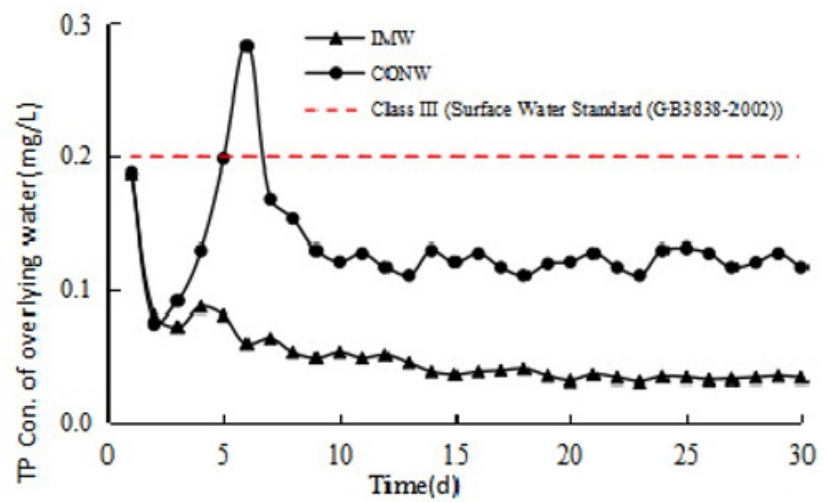

a

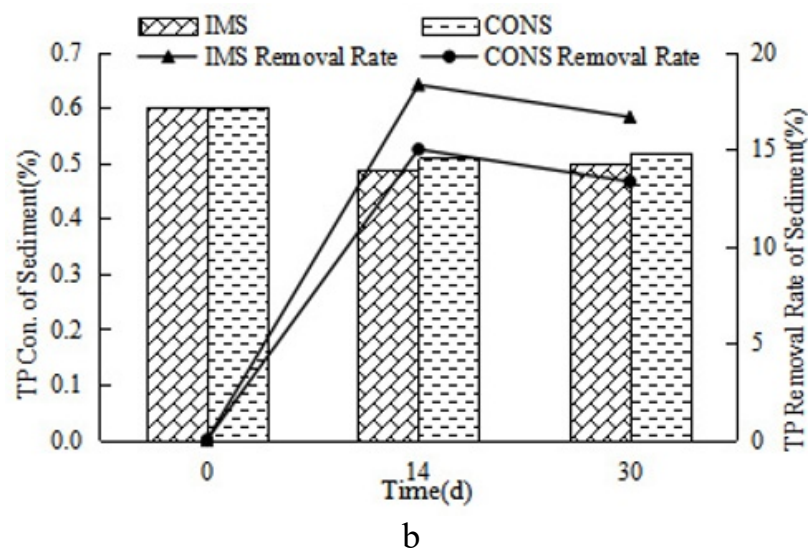

Fig.4. Effect of immobilized microorganism on phosphorous pollutant

\subsection{Microbial diversity analysis}

In order to analyse microbial diversities and communities of the IM system, we took water and sediment from each tank and analysed it with high-throughput pyrosequencing. IMW(S) represented the water (sediment) taken from the IM system, $\mathrm{CONW(S)}$ represented the water (sediment) taken from the non-IM system, ORIW(S) represented the water (sediment) which was taken out before repairing. The community richness and diversity indices of ORI, CON and IM were shown in Table 1. There were higher values of ACE and Chao of sample IM than that of CON and ORI, indicating that the former had more richness than the others. The Shannon index of IM was higher than that the other two groups, which suggested that the microbial community in IM system was more diverse. The more richness of microbial population in IM system might be due to immobilized microbe in the IM group are gradually adapted to external environmental conditions, became a dominant species and mass reproduction. It also proved that the microorganisms in the system changed noticeably when repairing with immobilized microbe.

\subsection{Microbial community analysis}

The analysis of microbial structure is a significant way to understand the biodegradation capacities of biological treatment processes under different situations ${ }^{[6]}$. In some special environments metabolic potential of specific functional microorganisms plays an essential role in effective removal of pollutants ${ }^{[7]}$. The bacterial community compositions of samples at phylum level are presented in Fig. 5. The results for all samples show that there are a total of 53 identified bacterial phyla. The main phyla were Proteobacteria, Actinobacteria, Bacteroidetes, Chloroflexi, Euryarchaeota, Acidobacteria etc. The rest 47 phylas bacteria in the six samples were less than $5 \%$.

Table 1. Microbial community richness and diversity indices

\begin{tabular}{|c|c|c|c|c|c|}
\hline Sample & OTUS & Shannon & Chaol & ACE & Coverage/\% \\
\hline ORIW & 829 & 5.429 & 986.082 & 1015.082 & 99.3 \\
\hline IMW & 978 & 6.356 & 1134.024 & 1210.547 & 99.1 \\
\hline CONW & 874 & 6.344 & 898.864 & 936.159 & 99.6 \\
\hline ORIS & 1738 & 8.44 & 1890.5 & 1926.372 & 98.8 \\
\hline IMS & 2159 & 8.958 & 2362.911 & 2401.47 & 99.0 \\
\hline CONS & 2017 & 8.84 & 2185.509 & 2214.489 & 98.9 \\
\hline
\end{tabular}

the samples, their proportions were notably different. The most abundant phylum was Proteobacteria in all of the samples, which might be due to the phylum Proteobacteria made up the majority of the community composition of all samples, which is similar with previous studies ${ }^{[8-9]}$. Many bacteria involved in carbon, nitrogen, and sulfur cycling belong to Proteobacteria [10-11]. Furthermore the Proteobacteria can removes organic matter, especially in the waste water treatment system, and it also plays a major role in the composition of bacteria. The majority of Proteobacteria detected in all groups were considered to be symbiotic bacteria in aquaculture.

IMW was significant different from the ORIW and ORIW, CONW samples had the same subdominant microbial phylum Actinobacteria, IMW had 
Bacteroidetes as its subdominant microbial phylum. Altogether, these three phyla represented more than $90 \%$ of the total bacteria in the overlying water samples. The relative abundance of Bacteroidetes in IMW was higher than in ORIW and CONW. Bacteroidetes were well known for its closely related to the conversion of organic matter such as DNA, lipids and proteins ${ }^{[12]}$, the large number of Bacteroidetes bacteria in the IM group, indicating that there was a more active material cycle in the environment. By contrast, the ORIW, CONW samples had a larger number of Actinobacteria might be due to the main role of Actinobacteria is to promote the decay of the remains of animals and plants in the sediment. And there are more animals and plants remains in ORI system and CON system, so it is suitable for Actinobacteria survival.

When concerning the subdominant microbial phylum in sediment, compairing with the ORIS, Chloroflexi in CONS was increased while decreased in IMS. The less Chloroflexi in IMS idicating that it had a lower concentration of total organic carbon ${ }^{[13]}$. This was consistent with the lowest total organic carbon concentration as shown in Fig3b.

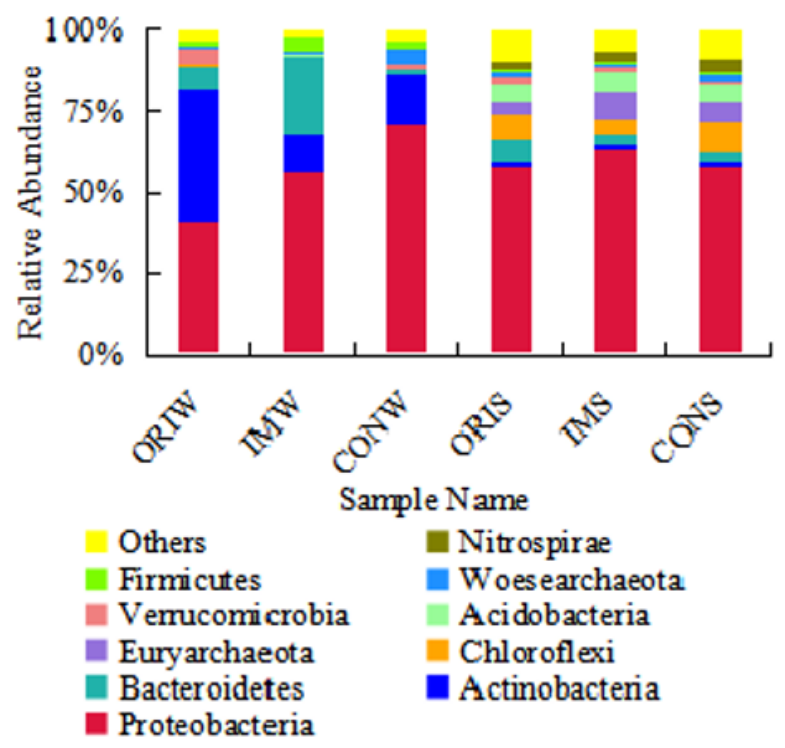

Fig. 5. Relative abundance of phylum-level bacterial communities in different samples

The structure of the microbial community in all samples was further investigated according to proportions of abundance at genus level, which is presented in Fig. 6. AS for sample IMW, most of the sequence reads were affiliated to five main genuses, namely Perlucidibaca, Limnohabitans, Bacillus, Hydrogenophaga, and Mycobacterium. Among them genus Bacillus are aerobic or facultative anaerobic bacteria. Its main feature is that each cell can produce elliptical or columnar endophytic spores, not inhibited by oxygen, and can resist many adverse environments ${ }^{[14]}$. AS for sample IMS, most of the sequence reads were affiliated to four main genus, namely Methanolinea, Methanoregula, Candidatus Competibacter, Methanosaeta. Among them genus Methanolinea and Methanosaeta can change acetic acid which was from the decomposition of organic decomposition in sediment into
$\mathrm{CH} 4, \mathrm{CO} 2$ or other gases, in order to achieve the reduction of sludge. ${ }^{[15]}$

After the remediation of immobilized microbial aeration technology, the dominant strains in water and sediment have changed greatly. The reason may be that the changes of physical and chemical factors in the environment play a role in the survival of the fittest. Changes in these floras may indicate and detect the changes before and after repairing, and have positive implications for stabilizing the internal functions of the ecosystem ${ }^{[16]}$.

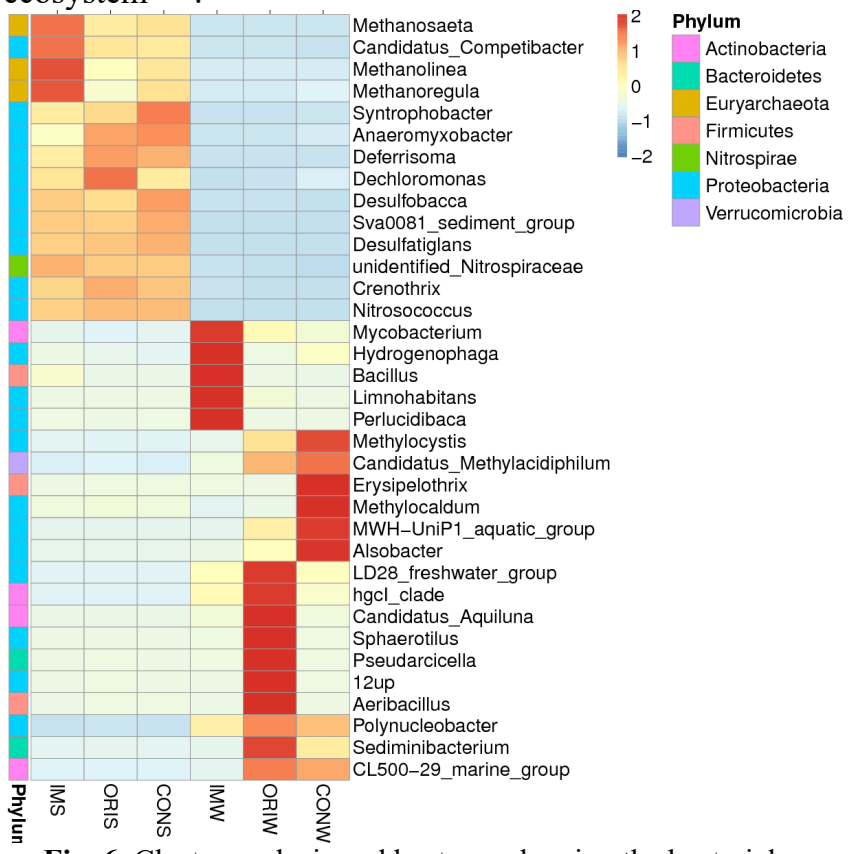

Fig. 6. Cluster analysis and heatmap showing the bacterial group classified at the genus level

The above analysis shows that the community composition of microbes is very sensitive to changes in environmental conditions, and previous studies have shown that the composition of bacterial communities is closely related to changes in environmental conditions [17-18]. In general, after the reparing, the bacterial community structure remained healthy and stable in the reactor, and the ecological environment was developed into a positive direction.

\subsection{Effect of environmental factors on bacterial community}

In order to explore the effect of environmental factors on microbial communities, based on the results of DCA

( Table S1), 10 dominant operational taxonomic units at phylum level for each sample were selected. Species-environment correlation represented the explained variance of each ordination axis to the species and cumulative percentage variance of the species-environment was used to denote the explained variance of environmental variables to species in RDA. Ordination results based on RDA were in Table S2. The correlation coefficient for the first and second ordination axis was 0 for both species and environmental factors, indicated that the ordination results were credible ${ }^{[19]}$. 
Table S1. The DCA analysis of 10 bacterial phylum in water samples

\begin{tabular}{|c|c|c|c|c|c|}
\hline Axes & 1 & 2 & 3 & 4 & Total inertia \\
\hline Eigenvalues & 0.134 & 0 & 0 & 0 & 0.227 \\
\hline Lengths of gradient & 0.738 & 0 & 0 & 0 & \\
\hline Cumulative percentage variance of species data & 59.2 & 0 & 0 & 0 & 0.227 \\
\hline Sum of all eigenvalues & & & & & 0 \\
\hline
\end{tabular}

Table S2. The RDA analysis of 10 bacterial phylum in water samples

\begin{tabular}{|c|c|c|c|c|c|}
\hline Axes & 1 & 2 & 3 & 4 & Total variance \\
\hline Eigenvalues & 0.697 & 0.303 & 0.000 & 0.000 & 1.000 \\
\hline Species-environment correlationst & 1.000 & 1.000 & 0.000 & 0.000 & \\
\hline $\begin{array}{c}\text { Cumulative percentage variance } \\
\text { of species data }\end{array}$ & 69.7 & 100.00 & 0.0 & 0.0 & \\
\hline $\begin{array}{c}\text { Cumulative percentage variance of } \\
\text { species-environment relation }\end{array}$ & 69.7 & 100.0 & 0.0 & 0.0 & 1.000 \\
\hline Sum of all eigenvalues & & & & & 1.000 \\
\hline Sum of all canonical eigenvalues & & & & & \\
\hline
\end{tabular}

Table S3. The DCA analysis of 10 bacterial phylum in sludge samples

\begin{tabular}{|c|c|c|c|c|c|}
\hline Axes & 1 & 2 & 3 & 4 & Total inertia \\
\hline Eigenvalues & 0.020 & 0 & 0 & 0 & 0.029 \\
\hline Lengths of gradient & 0.256 & 0 & 0 & 0 & \\
\hline Cumulative percentage variance of species data & 67.1 & 0 & 0 & 0 & \\
\hline Sum of all eigenvalues & & & & & 0.029 \\
\hline
\end{tabular}

Table S4. The RDA analysis of 10 bacterial phylum in sludge samples

\begin{tabular}{|c|c|c|c|c|c|}
\hline Axes & 1 & 2 & 3 & 4 & Total variance \\
\hline Eigenvalues & 0.838 & 0.162 & 0.000 & 0.000 & 1.000 \\
\hline Species-environment correlationst & 1.000 & 1.000 & 0.000 & 0.000 & 0.0 \\
\hline $\begin{array}{c}\text { Cumulative percentage variance of species data } \\
\text { species-environment relation }\end{array}$ & 83.8 & 100.00 & 0.0 & 0.0 & 1.000 \\
\hline Sum of all eigenvalues & 83.8 & 100.0 & 0.0 & 0.0 & 1.000 \\
\hline Sum of all canonical eigenvalues & & & & & \\
\hline
\end{tabular}

Water samples ORIW, CONW, IMW were located at different areas in the ordination diagram (Fig.7a), indicating they had different bacterial communities. All of the environmental factors were positively correlated to the first ordination axis. The Species-environment correlation coefficient for the first ordination axis was 1.0, and cumulative percentage variance of species-environment relation was $69.7 \%$ (Table S2), increased TP, COD, NH3-N and TN level, which increased bacterial richness in water. The second ordination axis negatively correlated with TP, COD, NH3-N and TN. Based on the length of connecting wire, TP had the largest impact on bacterial populations in water, followed by TP, COD, NH3-N and TN.

Correlation analysis of 10 dominant OTU at phylum level with environmental factors (Fig. 7b) showed that different bacterial phylum had different adaptability to water of different quality. As shown in Fig6b clese range indicated similar correlation for some bacterial phylum with water environment such as Acidobacteria (2), Nitrospirae (3), Bacteroidetes (4), firmicutes (5), the abundance of these four kinds of bacteria negatively correlated with all of the environmental factors. In addition Chloroflexi (1), Actinobacteria (9), Verrucomicrobia (10), also had a similar correlation, and their abundance were positively correlated with all of the environmental factors. There was no significant difference between the rest bacterial phylum and environmental factors.

In sediment samples, correlation between bacterial populations and environmental factors was similar with the result with water samples (Fig. 7c). The ORIS sample positively correlated with $\mathrm{TP}, \mathrm{TN}$ and TOC. While CONS sample and IMS sample were negatively 
correlated with them. The length of connecting wire showed that TP was the most influential environmental factor for bacterial populations in sediment samples, followed by TN and TOC.

Correlation of environmental factors with bacterial phylum in sediment samples was in Fig 7d. The abundance of Bacteroidetes (4), firmicutes (5),
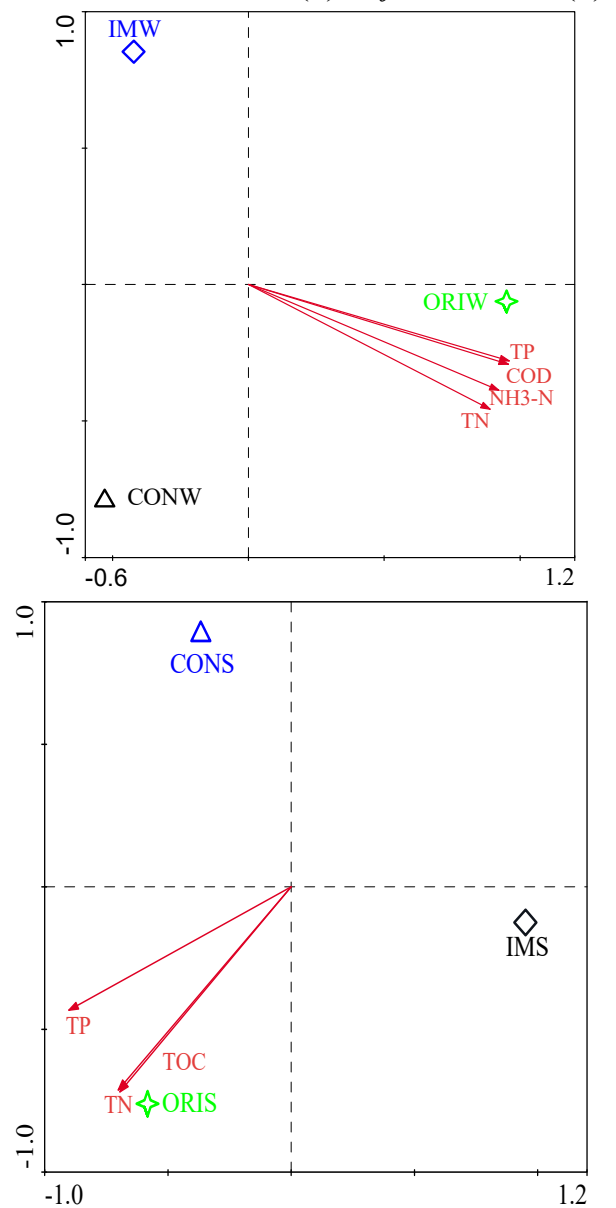

Actinobacteria (9), Verrucomicrobia (10), positively correlated with TP, TN and TOC. The correlation of Acidobacteria (2), Nitrospirae (3), Proteobacteria (6), Euryarchaeota (7) with sediment samples were positive. There was no significant difference between the rest bacterial phylum and environmental factors.
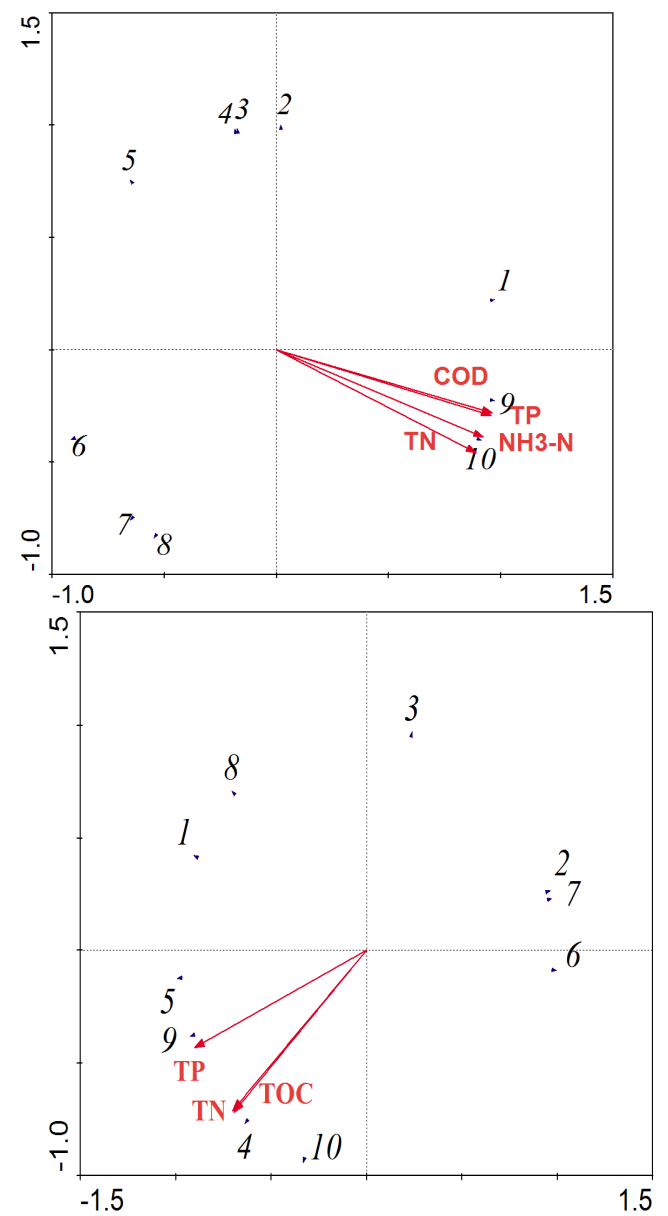

1. Chloroflexi, 2. Acidobacteria, 3. Nitrospirae, 4. Bacteroidetes, 5. Firmicutes, 6. Proteobacteria, 7. Euryarchaeota, 8.Woesearchaeota.DHVEG-6, 9. Actinobacteria, 10. Verrucomicrobia

Fig.7. RDA bioplot of distribution of the bacterial structure diversity and environment variables

\section{Conclusion}

(1)Immobilized microbe technology can degrade contaminants in the sediment and purify the overlying water. The polluted raw water, classified as inferior Class-V according to the surface water environment quality standard (GB3838-2002), upgraded to Class-III in 30-days remediation with TOM, TN and TP removal rates of $33.35 \%, 43.35 \%$ and $23.20 \%$, respectively. In contract, the control group without the assistant of aerated immobilized microbe still remains at Class-IV.

(2)High-throughput sequencing analysis reveals that immobilized microbe technology could improve the species diversity and abundance of the flora, and enhance the similarity between the sediment and the overlying water in the system.

(3)High-throughput sequencing analysis reveals that the dominant bacteria of the overlying water were Perlucidibaca, Limnohabitans, Bacillus,
Hydrogenophaga, Mycobacterium etc. And the dominant bacteria of the sediment were Methanolinea, Methanoregula, Candidatus Competibacter, Methanosaeta etc.

(4)Redundancy analysis shows that total phosphorous is the key environmental factor of microbial community structure in both overlying water and sediment.

\section{References}

1. Junter G-A, Jouenne T. Biotechnol Adv, 22 (8): 633658. (2004)

2. Bowman JS, Rasmussen S, Blom N, Deming JW, Rysgaard S, Sicheritz- Ponten T. Isme J, 6, 11. (2012)

3. Pitta DW, Pinchak WE, Dowd SE, Osterstock J, Gontcharova V, Youn E, Dorton K, Yoon I, R. Min B, J. D. Fulford, A. Wickersham T, P. Malinowski D. Microb Ecol, 59: 511-522. (2010) 
4. Zhu S, Huang X, Ho S H. Bioresource Technolo, 229:196-203. (2017)

5. Wen Li-li, Ye Zheng-fang, Ni Jin-ren. Journal of Basic Science and Engineering, 16(1):12-22. (2008)

6. Jiang Y, Wei L, Zhang H, et al. Bioresource Technol, 218:146.(2016)

7. Kormas KA, Pachiadaki MG, Karayanni H, Leadbetter ER, Bernhard JM, Edqcomb VP. Extremophiles, 19(5): 949-960. (2015)

8. Peralta R M, Ahn C, Gillevet P M. Sci Total Environ, 443(3), 725. (2013)

9. Zhong F, Wu J, Dai Y, Yang L, Zhang Z, Cheng S, Zhang Q. Appl Microbiol Biot, 99(3): 1499-1512. (2015)

10. Ansola G, Arroyo P, Le S D M. Sci Total Environ, s 473-474(3): 63-71. (2014)

11. Liu J, Yi N-K, Wang S, Lu L-J, Huang X-F. Ecol Eng, 94:564-573. (2016)

12. Cottrell M T.Kirchman D L. Appl Environ Microb,
66(4):1692-1697.(2000)

13. Zhao D, Rui H, Jin Z, Yan W, Wang J, Ma T, Wang M, L.Wu Q. World $J$ Microb Biot, 28(11):3159-3170. (2012)

14. Zhang Ji-zhong. Shanghai: Fudan University Press, 44-45. (in Chinese) (1999)

15. Sun Xing-fu. Chongqing University, 2013. (in Chinese) (2013)

16. M. S. Girvan, Campbell C. D, Killham K, Prosser J. I, Glover L. A. Environ Microbiol, 7(3):301-13. (2005)

17. Payne M S, Hall M R, Sly L, Bourne DG. Appl Environ Microb, 73(6): 1940-1951. (2007)

18. Zeng Y H, Ma Y, Wei C L, Jiao N Z, Tang K X, Wu Z H, Jian J C. Aquac Res, 41(9): e172-e186. (2010)

19. Zhang H, Sun Z, Liu B, Xuan Y, Jiang M, Pan Y, Zhang Y, Gong Y, Lu X, Yu D, Kumar D, Hu X, Cao G, Xue R, Gong C. Aquaculture 2016a, 455: 97-108. (2016) 